\title{
Rip It Up and Start Again: The rejection of a characterization of a phenomenon
}

Abstract: In this paper, I investigate the nature of empirical findings that provide evidence for the characterization of a scientific phenomenon, and the defeasible nature of this evidence. To do so, I explore an exemplary instance of the rejection of a characterization of a scientific phenomenon: memory transfer. I examine the reason why the characterization of memory transfer was rejected, and analyze how this rejection tied to researchers' failures to resolve experimental issues relating to replication and confounds. I criticize the presentation of the case by Harry Collins and Trevor Pinch, who claim that no sufficient reason was provided to abandon research on memory transfer. I argue that skeptics about memory transfer adopted what I call a defeater strategy, in which researchers exploit the defeasibility of the evidence for a characterization of a phenomenon.

Keywords: scientific phenomenon; evidence; defeasibility; memory transfer

\section{Introduction}

The identification of phenomena is a critical scientific research activity, as it is responsible for the discovery and characterization of the types of events to be explained by theory. To fulfill their theoretical and practical aims, researchers set out to accept characterizations of phenomena when empirical findings are put forward in their favor. When a characterization of a phenomenon is accepted, researchers theorize and experiment in a way that is consistent with the existence of the phenomenon. However, many episodes in the history of science involve the abandonment of characterizations of phenomena that were once empirically promising. This raises a question: under what circumstances do researchers reject a characterization of a scientific phenomenon, despite evidence that appears to support it?

In this paper, I analyze the rejection of a phenomenon through the lens of two philosophical topics. The first topic relates to how empirical findings can serve as evidence for a characterized phenomenon, and the defeasible nature of this evidence. The second topic relates to the strategies through which researchers test an existing characterization 

characterization of a phenomenon. By defeating all evidence, any empirically motivated reason for accepting a phenomenon as characterized is eliminated. With this strategy, researchers do not simply provide evidence to challenge a characterization of the phenomenon; they also demonstrate the faultiness of the experiments whose findings are thought to support the characterization.

I explore a case in which the characterization of a phenomenon was rejected: the research on memory transfer. This alleged phenomenon was described as the transfer of learned behavior by the insertion of tissue from a trained donor organism to an untrained receiver. It received a great deal of attention from scientists and the public alike, due to its implications and to researchers' use of sensational experiments involving cannibalism. Formulated and defended in light of empirical findings, the characterization of memory transfer was considered by some to be accurate; this led to a cottage industry about its characterization, its theoretical significance, and its underlying mechanisms. The research program was abandoned, and contemporary scientists generally consider the "phenomenon" to not exist.

The case of memory transfer has generated controversy in the history and philosophy of science. Sociologists and historians of science have questioned the motives of the scientific community that abandoned research on memory transfer. For instance, Harry Collins and Trevor Pinch argue that there was no "decisive technical evidence" that disproved the existence of memory transfer, and that research was abandoned due to disinterest in the purported phenomenon $(1998,25)$. Collins and Pinch present a powerful 
argue that there were deficiencies in the evidence put forward in opposition to memory transfer, and there is a discrepancy between the perceived decisiveness of evidence in opposition to memory transfer and the actual decisiveness of this evidence. They base their challenge on the fact that no evidence against memory transfer applies to all experiments whose findings were thought to provide support for the characterization of the alleged phenomenon.

Collins and Pinch's challenge is, at its core, one about the evidence required to reject a characterization of a phenomenon: they argue that there was no decisive evidence for the abandonment of research on memory transfer. I argue that the evidence provided against memory transfer was decisive for the rejection of the characterization of the alleged phenomenon. The only way to understand why there was decisive evidence, I argue, is to recognize the fact that scientific evidence is defeasible, and the defeat of evidence for memory transfer eliminated all reason to accept the characterization of memory transfer. By exploiting this fact, the defeater strategy provides a way to undermine evidence in favor of scientific claims, including characterizations of phenomena.

I will proceed as follows. In Section 2, I introduce the process of identifying phenomena, and discuss how empirical findings can serve as defeasible evidence for the characterization of a phenomenon. In Section 3, I present three projects in which researchers attempted to provide evidence for memory transfer. In 3.1, I examine the work of James McConnell on planarians. I continue in 3.2 with the development of memory research in mammals. In 3.3, I discuss the work of Georges Ungar. For each project, I review the dissenting opinions in the scientific community at the time. In Section 4, I 

be applied to other instances of scientific practice as well. With an account of this strategy, I rebut the claims about memory transfer presented by Collins and Pinch.

\section{Identifying a Scientific Phenomenon}

When researchers identify a scientific phenomenon, what is it that they identify? For this paper, a scientific phenomenon is a type of event whose characteristics exhibit repeatability and stability (Bogen \& Woodward 1988). This distinguishes phenomena from data, which are the empirical findings collected in experiments that are used to infer the characteristics of a phenomenon. Phenomena are discovered in the world or created in the laboratory (Hacking 1983, 221). Researchers aim to measure the features of phenomena that manifest in observational or experimental contexts, and use their empirical findings to accurately describe them by characterizing the features of their manifestations and the conditions under which they occur. Accepting a characterization of a phenomenon amounts to accepting that the phenomenon as characterized occurs. Conversely, rejecting this characterization amounts to rejecting that a phenomenon occurs in the way that it is characterized.

Characterizing phenomena is important for scientific practice. Theories are tested against the phenomena that are discovered or created; researchers seek to determine if the predictions they derive from their theories correspond to the phenomena they characterize (Bogen and Woodward 1988). In addition, characterizing a phenomenon "guides the 
construction of theoretical models (Craver \& Darden 2013, 52; see also Woodward 2003).

The identification of scientific phenomena is a process through which researchers discover and accurately characterize a phenomenon of interest. At the start, researchers record the features constitutive of a phenomenon from instances of its occurrence. To evaluate this initial characterization of a phenomenon, researchers produce the set of characterized features in the same and different contexts, to determine the conditions under which these features co-occur. These strategies allow the researchers to determine the features that constitute the phenomenon of interest, as well as the conditions that precipitate, inhibit, and modulate its occurrence (Craver \& Darden 2013, Chapter 4). When accurate, a characterization of a phenomenon corresponds to a general type of event, which occurs in the instances in which the experimental data were collected. If findings that support the characterization are produced, there is reason to accept that this phenomenon occurs and its characterization is accurate. This is because researchers take the findings to be causally related to the occurrence of an instance of the phenomenon.

Empirical findings provide evidential support for a characterization of a phenomenon, but this evidence is defeasible. Findings that serve as evidence for a characterization of a phenomenon do not entail its accuracy. The evidential status of findings may alter if additional findings are collected. Certain findings, known as defeaters, provide reason to think that the initial findings do not provide evidence for the characterization of the phenomenon. If evidence is defeated, it no longer provides reason to accept the characterization of a phenomenon. This depiction of defeasibility hints at a strategy that researchers can adopt: if researchers sever the relationship between findings 
for, they provide reason to no longer accept it. Undefeated evidence is required to accept a characterization of a phenomenon, and researchers can actively seek to defeat evidence in order to provide reason to reject it. This strategy, which I call the defeater strategy, played a crucial role in the abandonment of research on memory transfer.

An alternative strategy to provide reason to reject a characterization of a phenomenon is to develop new experiments whose findings provide evidence of the inaccuracy of the characterization. However, this strategy presents an additional challenge: evidence against a characterization of a phenomenon does not cause any evidence for it to lose its epistemic status. To dismiss findings as evidence for a characterization of a phenomenon, researchers must formulate an alternative hypothesis, inconsistent with the characterization of a phenomenon in question, which is better supported by the sum of the reported findings. In research on memory transfer, this kind of strategy was not used. This is because, as I will illustrate, there is no single reason why researchers produced findings they thought supported memory transfer as characterized.

\section{Memory Transfer}

Research on memory transfer developed out of research on learning in planarian worms in the 1950s, with a report of a transfer effect in 1962. By the end of the 1970s, most researchers agreed that the phenomenon did not exist. In this section, I track memory transfer research through three research projects (see Appendix for a table of articles that support or challenge memory transfer). Rather than present a strict temporal ordering of the projects, I present them based on their research questions and character of 
then discuss research on mammals by the Fjerdingstad group, the Jacobson group, and the Rosenblatt group. I finish by discussing Ungar's research on memory transfer and its underlying mechanism. I do not intend to provide an exhaustive history of the case. Rather, I focus on the researchers who contemporaries and historians agree played the most significant role in the discovery, characterization, theorization, explanation, and defense of memory transfer, and demonstrate how, in each set of research projects, there were unresolved experimental issues (Irwin 1978; Travis 1981; Setlow 1997).

The research on memory transfer was driven by experiment. The major proponents of memory transfer were experimentalists, who did not develop a characterization of the phenomenon in light of a well-established theory of memory. Instead, research was based on speculations from earlier experimental findings. While the research was informed by background theory on memory, experiment, rather than theory, drove subsequent experimentation. Thus, rejecting memory transfer was not considered to entail the refutation of an otherwise successful theory that predicted its occurrence.

Each memory transfer experiment shares a protocol template. This template is as follows:

Donor Training: An organism is trained to display a behavior consistent with the association of stimuli, which indicates that the organism has learned.

Transfer from Donor to Receiver: Tissue is excised from the donor organism, and inserted into the receiving organism.

Receiver Training: The receiving organism is trained following a learning paradigm. Researchers determine if the receiver demonstrates the behavior consistent with having the memory, or learns more quickly than an organism that has not received tissue from the donor. 
Thus, in a memory transfer experiment, there must be an associative memory, and there

must be a transfer of that memory, which allows the receiver to do something that could not be achieved without having that memory.

Debate over memory transfer revolved around two kinds of issues with the experiments whose findings were thought to serve as evidence for the characterization of the phenomenon. The first was the failures to replicate the results. These failures occur when researchers are unable to reproduce the findings reported by the supporters of the alleged phenomenon, despite reproducing the reported conditions of the original experiment. The second was the identification of confounds. In the present context, a confound is any feature not included in the characterization of the phenomenon that plays a role in producing the empirical findings in the phenomenon's purported experimental demonstration.

\subsection{McConnell and the Worms}

Research on memory transfer developed from research on the planarian as a model organism for neural experimentation. With Robert Thompson, McConnell putatively demonstrated that planarians could be classically conditioned (1955). To demonstrate conditioning, an organism must display behavior consistent with the association of an unconditioned stimulus and a conditioned stimulus, following tandem presentation of the stimuli to the organism. This amounts to the organism displaying a behavioral response to the unconditioned stimulus when the conditioned stimulus is presented. Thompson and McConnell had the unconditioned and conditioned stimuli played by electrical shock and light exposure respectively: each planarian was trained to react to light exposure, with its 

simplest organisms known to be able to do this.

The conditioning paradigm filled the Donor Training step of the protocol template. In the study, the planarians were divided into four groups: An experimental group (which received conditioned and unconditioned stimuli), a light control group (which received only conditioned stimulus), a shock control group (which received only unconditioned stimulus), and a control group (which received neither stimulus). Following their exposure to the stimulus (or lack thereof), the researchers observed the behaviors of the planarians. Only members of the experimental group demonstrated a significant increase in the number of behavioral responses. Organisms in this group received a three-second light stimulus, in which their behavior was recorded during the first two seconds, and they were shocked during the last second. That they engaged in the behaviors associated with the shock when exposed to the light only suggests that they had learned to associate the stimuli.

With a learning paradigm developed, McConnell began to investigate if a planarian would retain its memory following segmentation and regeneration of its body (McConnell 1965). Planarians regenerate if cut transversely: each half will grow back a head or tail to result in two distinct bodies. The researchers determined that if the planarians were trained to demonstrate the conditioned response, then cut in half and allowed to regenerate, each new planarian demonstrated the conditioned response (McConnell, Jacobson, \& Kimble 1959). This was considered remarkable. Neural structures are largely absent in the planarian tail, and were regrown after segmentation; it was "as if their new 
Please cite Final Version - Colaço, D. (2018). Rip it up and start again: The rejection of a characterization of a phenomenon. Studies in History and Philosophy of Science 72, 32-40.

brains were created with the old 'learning' already 'wired in'” (McConnell 1965, 6). These

findings sparked considerable debate, and led McConnell to develop a journal dedicated to planarians, called the Worm Runner's Digest. Though the journal included poems, comics, and facetious articles as part of McConnell's desire to introduce zaniness into an austere scientific community (Travis 1981), the journal also included serious papers, including the article that presented the first characterization of memory transfer.

If planarians could regenerate memories, McConnell speculated that 'the 'engram' must be stored throughout the planarian's body", and these 'engrams' might be transferrable between organisms (McConnell 1965, 6). To determine if transference was possible, he developed an infamous protocol that exploited the cannibalistic nature of planarians:

We trained some 'victim' worms to criterion (using the now-standard light/shock conditioning technique). We then fed the trained victims to starved, untrained cannibals. At the same time, a set of untrained 'victim' worms was fed to a different group of cannibals. Both sets of cannibals were then given light/shock training. The results were clear-cut: the cannibals that had ingested 'trained' victims were, on the very first day of training, significantly superior to the cannibals that had eaten untrained animals (McConnell 1965, 7).

First published in the Worm Runner's Digest in 1961, then in the Journal of Neuropsychiatry in 1962, 'Memory transfer through cannibalism in planarians' includes the first characterization of memory transfer: "Learning seems to be transferrable from one animal to another via cannibalistic ingestion" (McConnell 1962, S48). The characterization includes specific features of the experiment, but indicates the generalizability of the type of memory event. Through the cannibalistic transfer of tissue, the memories of the donor organism are transferred to the receiving organism. 
The characterization of the phenomenon and the learning paradigm both drew

controversy. Failures to replicate the learning in planarians were reported (Bennett \& Calvin 1964). Confounds were also reported. Researchers suggested that McConnell may not have demonstrated associative learning, and that the protocol instead may provoke the response of sensitization (Halas, James, \& Stone 1961). Sensitization is non-associative, so it is not the kind of learning McConnell had depicted in his characterization of memory transfer. If light provoked a response - a circumstance McConnell attempted to control for with the light control group - then the experiment would not have shown the association of the stimuli. Skeptical researchers found "a significant difference between [the light control group] and [the normal response control group]" (Halas, James, \& Knutson 1964, 791). They state that there is "no adequate explanation for this discrepancy other than to point out that there was a trend toward a significant difference between these groups in the Thompson and McConnell study": they point out that McConnell's 1962 article references a non-significant increase in behavioral responses in the light control group, suggesting that planarians may be responding to light as though it were an unconditioned stimulus (Halas, James, \& Knutson 1964, 791).

Other researchers criticized McConnell's protocol in his 1962 article. Researchers demonstrated a significant relationship between the shocking of planarians and their light response, but that this relationship was due to the fact that the cannibal planarians had ingested shocked tissue (Walker \& Milton 1966). Researchers were able to demonstrate behaviors consistent with McConnell's results, without training the donor worms. This criticism was extended by research in which conditioning was extinguished - that is, the association was formed through training and then dissociated through further training 
(Walker 1966). There was no significant difference in the behavior between the worms

that had consumed tissue from conditioned planarians, when compared to those that had been conditioned and then extinguished.

Thus, McConnell's research was undermined for two reasons. First, his findings had failed to replicate, raising concerns for the characterization of McConnell's experimental conditions. As critic Edward Bennett states, no one could "point to a $100 \%$ procedural replication", which made it "impossible in the absence of further experiments to determine if the relevant factors necessary for reproducible and reliable training of planarians have been described" $(1970,150)$. Second, several research groups provided reasons to suspect the protocol did not have the sophistication to rule out confounds like sensitization. This meant that McConnell's experimental findings were equivocal between demonstrating memory transfer and demonstrating an effect of a very different kind. Consequently, empirical challenges defeated the evidence McConnell provided for his characterization of memory transfer.

\subsection{The Inclusion of Mammals and The Theorization of Memory Transfer}

Following popularization by McConnell, other researchers became interested in memory transfer. Respecting the issues of McConnell's experiments, rodents were used "to avoid any discussion of whether... [the] experimental subjects could learn", and to determine if "the phenomenon would prove so general as to be found in mammals" (Fjerdingstad 1971, xvi-xvii). Following the transition to mammals, several research groups putatively demonstrated memory transfer. However, given that a principal question posed to researchers was "Does the phenomenon really exist...?", it is clear that 
Rosenblatt's group.

Following up on a pilot study that appeared to demonstrate memory transfer, researchers in the laboratory of Enjar Fjerdingstad developed a protocol to investigate transfer in mammals (Nissen, Røigaard-Petersen, \& Fjerdingstad 1965). Rats were trained via a reinforcement paradigm, meaning that the organisms behave consistently with the association of a behavior and a reward stimulus. Following training, their brains were extracted using a phenol solution, chemically treated in an isopropanol solution, and injected into receiving organisms. The chemical treatment increased the concentration of the RNA from the tissue, a possible chemical substrate for memory transfer. Their experiment followed the protocol template: donor rats were conditioned either to light or to darkness. Receiving rats were injected with tissue from light-conditioned or darkconditioned rats, and then were trained to reinforce dark preference or light preference. The publication reports a significant difference between the learning quickness of rats injected with light-condition donor tissue and those who were injected with darkconditioned donor tissue. However, the direction of difference is the opposite of what was predicted: light-conditioned rats gave better performance under reinforcement of dark preference and vice versa. This 'reversal effect' is inconsistent with the association of behavior and reward. Individual differences of rats and "specific inhibitory effects" are cited as potential explanations for the reversal effect (Nissen, Røigaard-Petersen, \& Fjerdingstad 1965, 271). 
Concurrent with this research, members of the laboratory of Allan Jacobson began

to investigate memory transfer. The researchers used a Skinnerian training paradigm to teach rats to associate clicking sounds with a behavior: rats would approach a food bowl when a click occurred, even if there was no food in the bowl (Babich et al. 1965). Once learning was established, the rats' brains were extracted and then injected into rats in an experimental group. The researchers also included a control, with untrained donor chemicals being injected into a group of rats. When comparing the behavior of the experimental and control groups, there was a significant difference in the tendency the rats had to approach the bowl when the clicking occurred. This study was reproduced, and the findings were replicated. The receiving organisms did not need to undergo training to display the behavior; they simply 'had' the memory following the injection.

Researchers in the laboratory of Frank Rosenblatt also began to study memory transfer. Reproducing Jacobson's protocol, Rosenblatt and colleagues reproduced the experiment and replicated the findings consistent with memory transfer (Rosenblatt, Farrow, \& Herblin 1966a). In addition to a direct replication, the researchers also modified the Donor Training step of the protocol template, and showed that associations formed by different training paradigms could be transferred. However, the researchers also uncovered individual differences within their subject population. They state that "it appeared from these data that the injection had 'taken' on some of the rats, but not on others" (Rosenblatt, Farrow, \& Herblin 1966b, 48). In another replication of Jacobson, the laboratory researchers confirmed issues with the injection 'taking': "we find repeated examples of this phenomenon", and, though "the reasons... are still subject to speculation", the authors include variation of dosage, subjects' sensitivity to the extract, and "the transfer 

behavior (Rosenblatt, Farrow, \& Rhine 1965, 553-554).

Despite complications, Rosenblatt states that his experiments convinced him "that the phenomenon of 'memory transfer' is a real one, which must be taken into account in any theoretical approach to biological memory" $(1967,34)$. However, given the lack of evidence about the chemistry that underlies transfer, he suggests that "it must be recognized that we are, in fact, entering the realm of science fiction; the present experiments, although suggestive, leave us completely in doubt as to the mechanism at work" (Rosenblatt 1967, 34). Thus, Rosenblatt accepted the characterization, even with no knowledge of what mechanistic details might underwrite memory transfer's occurrence. With no evidence for the mechanism, Rosenblatt admits that any effort to model what causally underwrites memory transfer amounts to speculation. This frustrated more eager modelers, with one saying that "we are inclined to feel that it is no more science fiction than was, for example, Einstein's derivation of his famous $E=m c^{2}$ relation at a time when there was not a single shred of experimental evidence for it" (Rashevsky 1968, 342). Modelers who were convinced of memory transfer modeled it, and assumed that subsequent research would vindicate their models.

The empirical results of these groups were questioned during this time. In an 18experiment replication performed by researchers in a number of different laboratories, there was failure to replicate the memory transfer phenomenon in a direct reproduction of Jacobson's protocol (Byrne et al. 1966). The failures suggest complications with Jacobson's protocol - and, by implication, Rosenblatt's protocol - though the authors state that "it 

pursuit of" memory transfer, as "failure to reproduce results is not, after all, unusual in the early phase of research when all relevant variables are as yet unspecified" (Byrne et al. 1966, 658). Thus, the failures to replicate provided reason to think that Jacobson's findings could not serve as evidence for memory transfer. However, those who worked on memory transfer continued to iterate their practices, to develop an experiment that did not include confounds and could be replicated.

Responding to the confounds identified in their previous work and the failures to replicate, Fjerdingstad and colleagues modified their protocol "in order to make the 'transfer effect' more reproducible", which was "considered to be of primary importance before further experiments concerning the degree of specificity of this effect and [its] exact chemical nature" (Røigaard-Petersen, Nissen, \& Fjerdingstad 1968, 1). To control for the individual differences of the organisms, the researchers picked only rats that were explorative. Fjerdingstad and colleagues developed a novel light-dark reinforcement experiment. Prior to injection, the researchers kept the chemicals at consistent temperatures, to control for any damage that may occur to the chemical under improper holding conditions. While the researchers report some effect on learning from the injection of control organisms - those injected with tissue from donors who were not trained - when compared to noninjected ones, they report a significant difference between the learning of the experimental and control injected groups.

However, the researchers replicated the reversal effect - the rats with the dark reinforcement injections demonstrated improved light behavior, and vice versa - which is likely why the researchers mention that their findings "must therefore be interpreted with 
Please cite Final Version - Colaço, D. (2018). Rip it up and start again: The rejection of a characterization of a phenomenon. Studies in History and Philosophy of Science 72, 32-40.

some reservation until these relations have been further investigated" (Røigaard-Petersen,

Niseen, \& Fjerdingstad 1968, 12). The researchers again cite individual differences of the

experimental organisms and inhibitory effects as potential explanations for the reversal

effect. They did not rule out confounds related to inhibitory effects, even though their

results suggest that something that is not specified in the characterization of memory

transfer likely played a role in their experiments.

In response to the failures to replicate, the researchers state the following:

During the last year the proportion of the number of 'positive' to the number of 'negative' reports seems to have changed. No doubt this is due to the realization of the potential importance of even seemingly trivial variables, both of behavioral methods, extraction and injection. Too little attention to these factors characterize [sic] many of the early negative reports (RøigaardPetersen, Niseen, \& Fjerdingstad 1968, 14).

While the researchers do not make clear which factors the negative reports missed, their intent is clear: negative reports, but not positive ones, fail to take into account key experimental features. It is due to this issue with the negative reports, they suggest, that replication does not occur.

Thus, the three laboratory groups were unable to resolve issues relating to replication failures or confounds. Many were unable to replicate the findings presented by the supporters. This was summed up in the comments from one critic that "it is essential that several laboratories can replicate and extend the primary observations" (Bennett 1970, 150). Critics did not accept the speculation that confounds were responsible for only failures to replicate. Furthermore, the supporters did not address which features of their experiment led to the inconsistent findings presented by Fjerdingstad and colleagues. For these reasons, the findings were not taken by the community to evidentially support the characterization of memory transfer. 


\subsection{Ungar, Mammals, and the Mechanism of Memory Transfer}

One other researcher was a key investigator of memory transfer in mammals.

Georges Ungar started working on memory transfer with the investigation of the transfer of habituation from mice to rats. In this experiment, the Donor Training involved exposing the donor organisms to a stimulus that would elicit a startle response in the organism (Ungar \& Oceguera-Navarro 1965). Once the donor organisms were acclimated to the stimulus, they were killed and their tissue was transferred. Along with this, a control group was injected with the brains from naïve organisms. Following injection, the researchers report a significant difference in the habituation rates of the two groups, with the experimental group habituating much more quickly. This led Ungar to investigate associative learning.

In his research, Ungar typically exposed the experimental injection to a battery of chemical reactions, and, from this, determined that a key component of the injection was a protein or peptide chain. As such, he took failures to replicate memory transfer (such as Byrne et al. 1966) to be irrelevant to his work, as Byrne and colleagues did not concentrate peptides. However, he explained the previous successes by Jacobson's group to be due to poor design, and the failure to remove peptides from their injection.

Ungar continued to perform experiments to determine the specificity of the transfer of associated memory. With a publication that summed up his research, Ungar concluded that he had produced sufficient evidence for the phenomenon's existence (Ungar 1970). The features relating to chemical composition, dose, interval between injection and testing 
had not been controlled for in failures to replicate. However, Ungar also states:

The reliability of the method is limited because of the multiplicity of the factors determining success and failure. The experience accumulated in the last five years in many laboratories explains most of the past failures (1970, 162).

This hedging of claims about memory transfer came at the same time as another series of failures to replicate: one in a new experimental context (Krech \& Bennett 1971), and the other a direct replication of Ungar's protocol (Goldstein, Sheenan, \& Goldstein 1971). With critics demanding "an experimental procedure which will yield consistently positive results in the hands of all qualified experimenters and in different laboratories", skeptics would not settle for "weak positive answers, only fitfully obtained, and only among a chosen few experimenters" (Krech \& Bennett 1971, 161). For skeptics to endorse Ungar's results, he had to produce a more sophisticated experiment, or else modify his characterization of memory transfer.

To convince the skeptics, Ungar developed a large sample study to demonstrate the phenomenon (Ungar, Desiderio, \& Parr 1972). Training rats and mice on dark avoidance, the project involved 4000 experimental subjects trained on the paradigm, whose brains were then prepared to isolate a peptide. Ungar believed this peptide to underwrite associative memory, and further believed its isolation and transmission from one organism to another via injection to be the mechanism underwriting memory transfer. The researchers report a significant difference in the dark-avoidance behaviors of the experimental group from both the group that received injections from untrained donors, and those who received no injections at all. Individual differences between the subjects are not reported, nor are controls on the experimental protocol except when relating to the 
with previous memory transfer experiments.

Ungar's 1972 article was published with commentary from the reviewer Walter Stewart, as Ungar was not able to satisfy Stewart's reservations with the experiment. Stewart agreed that Ungar demonstrated the synthesis of the peptide that could potentially causally underwrite the alleged phenomenon, but that isolation of the brain material from the rodents was "so grave... that the authors' conclusions are more likely false than true" (Stewart 1972, 209). Furthermore, Stewart raises concerns about whether or not "the bioassay [can] be successfully repeated outside of [Ungar's] laboratory", and what conditions are "necessary to minimize its variability" (Stewart 1972, 209). In his commentary, Stewart challenges Ungar to reproduce the phenomenon and have other laboratories successfully use his protocol. He also mentions that Ungar's protocol is quite different from previous research, resulting in a worry that Ungar has not demonstrated if his work relates to previous successes and failures to demonstrate memory transfer. Ungar was never able to meet Stewart's challenge. He was never able to develop a protocol in which he or anyone else could consistently demonstrate memory transfer. This failure, in result, led researchers to not accept that he had provided evidence for the alleged phenomenon.

\subsection{Aftermath}

No supporter of memory transfer was able to provide a clear characterization of the phenomenon and the conditions under which it occurs. While modelers theorized about the phenomenon's implications, and experimentalists investigated its underlying 

states, "it was not confusion over the biochemical identity of the transfer factors or the debate over the behavioral specificity of the effect which most damaged the credibility of the transfer paradigm, but simply the unreliability of the phenomenon" $(1978,486)$. Thus, it was not an issue with determining the mechanistic underpinnings of memory transfer that doomed the research program; it was the persistent defeat of any evidence for the alleged phenomenon. Irwin goes on to say, "the phenomenon was by no means universally replicable, and many labs... failed to obtain satisfactory evidence for any form of transfer", and "even for the proponents of the transfer phenomenon, the magnitude of the effect was always marginal" $(1978,486)$.

McConnell turned out to be right that planarians can learn (Rilling 1996), though his protocol was not sophisticated enough to demonstrate it. Those working on the biochemistry of memory started again, to investigate memory without the baggage of memory transfer. Progress in this field was made, but later researchers did not return to the memory transfer research of the 1960s and the 1970s. Today, most researchers think that the study of memory transfer was flawed (Setlow 1997), and any transfer phenomenon that might exist would likely have a very different characterization.

\section{Rejecting the Characterization of a Phenomenon}

Issues with the experiments whose findings were thought to provide support for the characterization of the purported phenomenon precipitated the abandonment of the investigation of memory transfer. In this section, I analyze what issues like these indicate 
Please cite Final Version - Colaço, D. (2018). Rip it up and start again: The rejection of a characterization of a phenomenon. Studies in History and Philosophy of Science 72, 32-40.

about the accuracy of a characterization of a phenomenon. I account for the evidential role of empirical findings in characterizing a phenomenon, and explain why many experiments were used to defeat evidence for the characterization of memory transfer.

\subsection{Why Did Researchers Reject the Characterization of the Phenomenon?}

Proponents of memory transfer faced the challenge of reported failures to replicate the finding when the reported conditions of the experiment were reproduced. In response, the supporters developed new experiments, but were unsuccessful: additional failures to replicate were reported, which themselves replicated. The supporters did not determine if the phenomenon occurs under a more constraining set of conditions than originally specified: there was no test of whether memory transfer only occurred in certain organisms, with certain learning paradigms, with only non-associative forms of memory, or with only certain associated stimuli. It may be the case that these variables must be held fixed for memory transfer to occur, and thus should have to be included in the characterization to delimit the conditions under which the phenomenon occurs.

Failures to replicate - meaning that researchers reproduce the reported conditions of the experiment, but not the result - suggest that there is issue with the characterization of the conditions under which a phenomenon occurs. The findings produced in the original experiment may be due to conditions that were not reported and not reproduced in the replication attempt. As a result, researchers infer that the characterization fails to specify the conditions that precipitate the phenomenon, or it fails to specify some conditions that inhibit it. As unexplained failures become more common, researchers' skepticism of the accuracy of characterization of the phenomenon grows, which is one reason why 

conditions under which the phenomenon may occur.

In addition, there was the issue of the identification of confounds in memory transfer research. These confounds were not thought to be constitutive of the phenomenon, but nevertheless appeared to be responsible for the findings thought to support the phenomenon's characterization. McConnell was unable to develop an experiment in which he could eliminate sensitization, Fjerdingstad was unable to eliminate the features responsible for the reversal effect, and Ungar was unable to eliminate individual differences between his experimental subjects. Skeptics developed experiments that suggested that these features might be responsible for the findings obtained in memory transfer experiments. McConnell, Fjerdingstad, and Ungar may have discovered real phenomena, but these phenomena were not accurately described by the characterization of memory transfer.

The identification of confounds reveals a problem with the characterization of the features thought to constitute the phenomenon. In normal circumstances, researchers take data, the empirical findings from individual experiments, to provide a basis for inferring a phenomenon (Bogen \& Woodward 1988). The occurrence of the phenomenon of interest is taken to be causally responsible for the data, and thus the data reflect the phenomenon's features. However, if there is a known confound that might be responsible for the findings, there is an alternative explanation for why researcher acquire the data that they do, undermining the inference from data to the characteristics of the phenomenon of interest. Researchers must determine whether the findings are indicative of the occurrence of the 
researchers cannot produce consistent findings while simultaneously eliminating the presence of the confound, the initial findings are equivocal.

In the memory transfer case, the skeptics produced findings consistent with those from McConnell's experiments without the involvement of memory. This trend continued in research on mammals: confounds were identified, sometimes by the supporters themselves. To restore evidential support to the characterization of the phenomenon, supporters had to either eliminate the confounds that had been identified, or recharacterize the phenomenon so as to not rule out that something aside from memory transfer may be responsible for their findings. The supporters were unable to do the former, and unwilling to do the latter. In general, as a result of the identification of actual confounds relevant to experiments in which positive results are reported, researchers' skepticism of the accuracy of the characterization of the phenomenon grew. This is another reason why memory transfer was abandoned. The characterization of memory transfer may have been too specific; it did not indicate that the phenomenon might involve the interaction of different sets of features, each of which was consistent with the experimental results.

Thus, findings from multiple experiments provided different insight regarding the accuracy of the characterization of memory transfer. The proponents of the phenomenon neither resolved the issues that were put forward, nor modified the characterization of memory transfer. With no experimental support and no independent theoretical support, there was no reason for members of the research community to accept the phenomenon. Thus, the characterization of memory transfer was rejected. 


\subsection{Why Were Researchers Justified in Rejecting the Characterization of the Phenomenon?}

The issues I have presented led researchers to reject memory transfer. However, one might question whether or not researchers were epistemically justified in their rejection. Collins and Pinch deny that there was sufficient reason to abandon memory transfer. They challenge the decisiveness of three articles they argue are cited as providing decisive evidence against memory transfer, each of which I mention in Section 3: the failure to reproduce planarian learning by Bennett and Calvin (1964), the failures to replicate by Byrne and colleagues (1966), and the reservations of Ungar's protocol by Stewart (1972). Collins and Pinch note that these publications "seemed decisive at the time", but "in retrospect they seem much less decisive" $(1998,24)$. Based on the evidential deficiencies of these articles, they conclude:

A determined upholder of the idea [of memory transfer] would find no published disproof that rests on decisive technical evidence. For such a person it would not be unreasonable or unscientific to start experimenting once more. Each negative result can be explained away while many of the positive ones have not been. We no longer believe in memory transfer but this is because we tired of it, because more interesting problems came along, and because the principal experimenters lost their credibility. Memory transfer was never quite disproved; it just ceased to occupy the scientific imagination (Collins \& Pinch 1998, 25).

While Collins and Pinch do not characterize what counts as a "disproof that rests on decisive technical evidence", their position can be inferred from their discussion of the limitations of the three articles. They note that each article targets only a subset of the empirical research on memory transfer. They claim that Bennett and Calvin's criticisms of planarian learning are moot, because it was later determined that planarians can learn, and their criticisms do not apply to experiments that involve model organisms that 
replicate presented by Byrne and colleagues are irrelevant to research by Ungar, because his protocols were different than those developed by the laboratory of Jacobson, which Byrne and colleagues investigated. They address no specific limitation of Stewart's article, but it can be inferred that their argument is based on the fact that Stewart's reservations only apply to issues with Ungar's experiments.

Collins and Pinch's conclusion can be read in two different ways. It may be the case that their argument rests on the fact that some evidence for memory transfer was not "explained away". However, this is inconsistent with the history of memory transfer research I have presented: every major experiment thought to provide support for memory transfer was questioned in light of the failures to replicate and the identification of confounds, and other reported experiments were based on the designs of the major ones. Alternatively, it may be the case that their argument rests on the fact that the criticisms expressed in each article apply only to certain experiments whose findings were thought to provide evidence for the phenomenon as characterized. No criticism applies to the total sum of empirical research on memory transfer. Those working with mammalian model organisms "explained away" the issues with planarian experiments. Ungar "explained away" the issues with experiments that did not involve the concentration of the peptides that he believed to underlie the memories that were transferred. It can be inferred that, to count as "disproof that rests on decisive technical evidence", researchers must demonstrate an issue that applies to all experiments whose findings are thought to provide evidential support for memory transfer. Alternatively, the skeptics must put forward an alternative hypothesis that is equally well supported by the proponents' empirical findings, and can be 

introduced in Section 2.

I agree with this reading of Collins and Pinch's argument: the challenges to the characterization of memory transfer targeted specific features of individual experiments, none of which can be applied to research in the field as a whole. Likewise, even though Collins and Pinch fail to discuss many articles critical of memory transfer, I agree that no critical publication describes an issue that applies to all memory transfer experiments, and no alternative hypothesis was presented that is equally well supported by the empirical findings. However, I disagree with the conclusion that Collins and Pinch draw from these facts.

Researchers were justified in their rejection of the characterization of memory transfer. Skeptics of memory transfer targeted the individual experiments that were used to produce the results that ostensibly demonstrated the phenomenon. The goal of the skeptics was to raise issues with each experiment whose findings were thought to provide evidence for memory transfer. Their strategy thus followed: reproduce the experiments, demonstrate issues with the experiments, and sever the evidential relationship between the findings from these experiments and the characterization of the phenomenon the findings were taken to support.

The skeptics reproduced the experiments in order to determine the relationship between the findings that were initially reported and the phenomenon's characterization. In the iterated experiments, they determined two kinds of things related to the issues in Section 4.1. First, they determined that the experiments did not produce findings 
Please cite Final Version - Colaço, D. (2018). Rip it up and start again: The rejection of a characterization of a phenomenon. Studies in History and Philosophy of Science 72, 32-40.

consistent with the features described in the characterization, despite the fact that the experiment was consistent with the conditions specified in the characterization. Second, they determined that the experiments involved features that could be responsible for the findings produced in the experiment, despite the fact that those features were not included in the characterization. Both issues suggest that the inference thought to be warranted by the findings from the original experiments - that they had demonstrated the phenomenon - is not warranted.

Aspects of the skeptics' strategy can be compared to the role of internalist epistemic defeaters in accounts of human reasoning and the analysis of knowledge. Epistemologists have claimed that knowledge claims are defeasible, meaning that the justification for a knowledge claim can be defeated by the acquisition of new evidence about the relationship between the claim and what is thought to justify it (Chisholm 1966). Likewise, cognitive scientists characterize the architecture of reasoning by determining the reasons individuals have for belief and the defeasibility of these reasons. A succinct characterization of defeaters in reasoning comes from John Pollock:

$\mathrm{R}$ is a defeater for $\mathrm{P}$ as a prima facie reason for $\mathrm{Q}$ if and only if $\mathrm{P}$ is a reason for $S$ to believe $Q$ and $R$ is logically consistent with $P$ but ( $P$ \& $R$ ) is not a reason for $\mathrm{S}$ to believe $\mathrm{Q} \ldots \mathrm{R}$ is an undercutting defeater for $\mathrm{P}$ as a prima facie reason for $S$ to believe $Q$ if and only if $R$ is a defeater and $R$ is a reason for denying that $P$ wouldn't be true unless $Q$ were true $(1987,484-485$, my emphasis).

If an individual believes a conclusion for a certain reason, an undercutting defeater is one that is consistent with the reason, but attacks "the connection between the reason and the conclusion" (Pollock 1987, 485). For example, the belief that an object is red because one perceives it to be red is defeated by the discovery that the object is illuminated by a red light. The undercutting defeater does not entail that the object is not red, but it is a reason 

conclusion.

The idea of undercutting defeaters can be applied to the strategy of characterizing phenomena. Researchers accept characterizations of phenomena upon the assessment of empirical findings that are taken to support these characterizations. This is because the occurrence of the phenomenon is thought to be responsible for the findings that researchers acquire. Undercutting defeaters undermine the evidential relationship between findings and a characterization. They provide reason to think that the occurrence of the phenomenon may not be responsible for the findings initially taken to support its characterization. Researchers who are skeptical of a phenomenon's characterization can actively seek to determine whether or not there are undercutting defeaters for the findings that are thought to support the characterization. To the extent that they can discover defeaters, they can undermine inferring the phenomenon from the empirical findings thought to support it. This is the defeater strategy.

Skeptical researchers employed the defeater strategy to systematically undermine all empirically motivated reason to accept the characterization of memory transfer. The undercutting defeaters in this case were the findings related to the failures to replicate, and the findings related to the identification of confounds. Each set of negative findings is an undercutting defeater for evidence for the characterization of memory transfer, as each provides reason to think that the features or conditions specified in the characterization of memory transfer may not be responsible for the experimental findings thought to support its characterization. Every major memory transfer experiment was challenged, and every 
reported positive finding was undercut by identified confounds and failures to replicate.

Thus, for every finding thought to provide evidence for memory transfer, an undercutting defeater was presented. Each defeater relates to a particular experimental attempt to demonstrate memory transfer. However, as a collection, the defeaters provide sufficient reason to think that there was no undefeated empirical evidence to accept the characterization of memory transfer. It was not merely the possibility of defeaters that undercuts evidence for the characterization of the memory transfer, as all empirical evidence is defeasible. It was the actual collection of findings that are undercutting defeaters of evidence for a characterization of memory transfer that plays this role.

The defeater strategy was appropriate in a case like memory transfer. This is due to the fact that many experiments were performed in the various memory transfer projects, whose findings were thought to provide evidence for memory transfer. The experiments were different, involving both differences in protocol and model organism. Skeptics suggested alternative explanations for the positive findings, but these explanations were sensitive to particular aspects of the protocol or organism involved. Thus, the issues related to McConnell's protocols were very different than the issues related to the work of Ungar, Jacobson, Rosenblatt, or Fjerdingstad. The fact that there was not a single factor that was responsible for all previous findings means that skeptical researchers were not able to develop an experiment that proved that any single factor, rather than the occurrence of memory transfer, was responsible for all findings. Instead, they challenged each experiment thought to demonstrate the phenomenon individually, and undercut every empirical finding thought to support its characterization. 
Collins and Pinch are right that it would not be unscientific for researchers to begin

again to search for memory transfer. Analogous to the fact that an undercutting defeater does not entail the falsity of the conclusion whose reason is undercut, defeaters do not rule out the possibility that there exists a phenomenon with features in the vague vicinity of what had been characterized. However, there is no reason to accept that the phenomenon occurs as characterized. More importantly, it would be unscientific to use the very same experiments described by supporters of memory transfer to rekindle investigation, due to issues with the reported conditions and identified confounds. It would take new techniques and protocols to renew the search for a phenomenon that is something like memory transfer.

The defeater strategy employed in research on memory transfer reflects a more general characterization of when researchers have reason to reject the characterization of a scientific phenomenon despite evidence that initially appears to support it. A characterization of a phenomenon ought to be accepted if there are empirical findings that provide reason to support it. If undercutting defeaters challenge the evidential role of the findings for the characterization of the phenomenon in question, and experiments that were thought to demonstrate the phenomenon are equivocal, then the empirical support for the characterization is reduced. If the empirical findings provide reason to accept the phenomenon as characterized, then it ought to be rejected if this reason is undermined. The effectiveness of the defeater strategy rests on challenging a characterization of a phenomenon by providing reason to think that the experiments whose findings are thought to provide support for the characterization are faulty, and lack the requisite sophistication to infer the occurrence of a phenomenon from its findings. More than providing evidence 
against the accuracy of the characterization of a phenomenon, this strategy provides a

means to challenge evidence put forward in the characterization's favor.

\section{Conclusion}

In this paper, I have analyzed an episode from the history of science in which there was a rejection of the characterization of a scientific phenomenon despite initially promising empirical findings. Proponents of memory transfer produced findings that were thought to support the characterization of the phenomenon. The issues with the experiments in which the findings were produced ultimately precipitated the rejection of the phenomenon as characterized. The experimental strategy employed by skeptics of the reality of the alleged phenomenon exploited the defeasible evidential relationship between the characterization of memory transfer that proponents accepted, and the empirical findings that served as reasons to accept it. My analysis of the memory transfer case provides a novel way to think about the assessment of scientific evidence. New experimental findings can defeat the evidence provided for a characterization of a phenomenon. This provides reason to reject the characterization, even if, as Collins and Pinch note, no one of the new experiments is individually decisive.

\section{References}

Babich, F., Jacobson, A., Bubash, S., \& Jacobson, A. (1965). Transfer of a response to naive rats by injection of ribonucleic acid extracted from trained rats. Science, 149(3684), 656-657.

Bennett E. (1970). Comments on The planarian controversy. In Molecular approaches to learning and memory (p. 151). 
Please cite Final Version - Colaço, D. (2018). Rip it up and start again: The rejection of a characterization of a phenomenon. Studies in History and Philosophy of Science 72, 32-40.

Bennett, E., \& Calvin, M. (1964). Failure to train planarians reliably. Neurosciences Research Program Bulletin, 2(4), 3-24.

Bogen, J., \& Woodward, J. (1988). Saving the phenomena. The Philosophical Review, 97(3), 303-352.

Byrne, W., Samuel, D., Bennett, E., Rosenzweig, M., Wasserman, E., Wagner, A., ... \& Fenichel, R. (1966). Memory transfer. Science 153, 635-636.

Chisholm, R. M. (1966). Theory of knowledge. Englewood Cliffs: Prentice-Hall.

Collins, H. \& Pinch, T. (1998). The golem: What you should know about science. Cambridge University Press.

Craver, C., \& Darden, L. (2013). In search of mechanisms: Discoveries across the life sciences. University of Chicago Press.

Fjerdingstad, E. (1971). Chemical transfer of learned information. Amsterdam: NorthHolland Publishing Co.

Goldstein, A., Sheehan, P., \& Goldstein, J. (1971). Unsuccessful attempts to transfer morphine tolerance and passive avoidance by brain extracts. Nature 211,11601163.

Hacking, I. (1983). Representing and Intervening: Introductory topics in the philosophy of natural science. Cambridge: Cambridge University Press.

Halas, E., James, R., \& Stone, L. (1961). Types of responses elicited in planaria by light. Journal of comparative and physiological psychology, 54(3), 302.

Halas, E., James, R., \& Knutson, C. (1962). An attempt at classical conditioning in the planarian. Journal of comparative and physiological psychology, 55(6), 969.

Irwin, L. (1978). Fulfillment and frustration: the confessions of a behavioral biochemist. Perspectives in biology and medicine, 21(4), 476-491.

Krech, D., \& Bennett, E. (1971). Interbrain information transfer: a new approach and some ambiguous data. In Chemical transfer of learned information (pp. 143-163). Amsterdam: North-Holland Publishing Co.

McConnell, J. (1962). Memory transfer via cannibalism in planaria. J. Neuropsychiat, 3, 1-42.

- - (1965). A manual of psychological experimentation on planarians. Ann Arbor: Worm Runner's Digest. 
Preprint Version: Published in Studies in History and Philosophy of Science David Colaço

Please cite Final Version - Colaço, D. (2018). Rip it up and start again: The rejection of a characterization of a phenomenon. Studies in History and Philosophy of Science 72, 32-40.

Nissen, T., Røigaard-Petersen, H., \& Fjerdingstad, E. (1965). Effect of ribonucleic acid (RNA) extracted from the brain of trained animals on learning in rats (II). Scandinavian journal of psychology, 6(2), 265-272.

Pollock, J. L. (1987). Defeasible reasoning. Cognitive science, 11(4), 481-518.

Rashevsky, N. (1968). Some possible theoretical implications of experiments on the chemical transfer of memory. Bulletin of Mathematical Biology, 30(2), 341-349.

Rilling, M. (1996). The mystery of the vanished citations: James McConnell's forgotten 1960s quest for planarian learning, a biochemical engram, and celebrity. American Psychologist, 51(6), 589.

Rosenblatt, F. (1967). Recent work on theoretical models of biological memory. In In Computer and Information Sciences-II. New York: Academic Press.

Rosenblatt, F., Farrow, J., \& Herblin, W. (1966). Transfer of conditioned responses from trained rats to untrained rats by means of a brain extract. Nature, 209(5018), 46-48.

Rosenblatt, F., Farrow, J., \& Rhine, S. (1966a). The transfer of learned behavior from trained to untrained rats by mean of brain extracts. I. Proceedings of the National Academy of Sciences, 55(3), 548-555.

- - . (1966b). The transfer of learned behavior from trained to untrained rats by means of brain extracts. II. Proceedings of the National Academy of Sciences, 55(4), 787-792.

Røigaard-Petersen, H., Nissen, T., \& Fjerdingstad, E. (1968). Effect of ribonucleic acid (RNA) extracted from the brain of trained animals on learning in rats (III). Scandinavian journal of psychology 9, 1-16.

Setlow, B. (1997). Georges Ungar and memory transfer. Journal of the History of the Neurosciences, 6(2), 181-192.

Stewart, W. (1972). Comments on the Chemistry of Scotophobin. Nature, 238, 202-209.

Thompson, R., \& McConnell, J. (1955). Classical conditioning in the planarian, Dugesia dorotocephala. Journal of comparative and physiological psychology, 48(1), 65.

Travis, D. (1981). On The Construction of Creativity: The 'Memory Transfer' Phenomenon and the Importance of Being Earnest. In The Social Process of Scientific Investigation (pp. 165-193). Springer Netherlands.

Ungar, G. (1970). Chemical transfer of learned behavior. Inflammation Research, 1(4), 155163. 
Please cite Final Version - Colaço, D. (2018). Rip it up and start again: The rejection of a characterization of a phenomenon. Studies in History and Philosophy of Science 72, 32-40.

Ungar, G., Desiderio, D., \& Parr, W. (1972). Isolation, identification and synthesis of a specific-behaviour-inducing brain peptide. Nature, 238, 198-202.

Ungar, G., \& Oceguera-Navarro, C. (1965). Transfer of habituation by material extracted from brain. Nature, 207, 301-302.

Walker, D. R. (1966). Memory transfer in planarians: an artifact of the experimental variables. Psychonomic Science, 5(9), 357-358.

Walker, D. R., \& Milton, G. A. (1966). Memory transfer vs. sensitization in cannibal planarians. Psychonomic Science, 5(7), 293-294.

Woodward, J. (2003). Making things happen: A theory of causal explanation. New York: Oxford University Press. 
Preprint Version: Published in Studies in History and Philosophy of Science David Colaço Please cite Final Version - Colaço, D. (2018). Rip it up and start again: The rejection of a characterization of a phenomenon. Studies in History and Philosophy of Science 72, 32-40.

\section{Appendix}

Table 1: Articles that support or challenge the characterization of memory transfer.

\begin{tabular}{|c|c|c|}
\hline Citation & Empirical Findings & $\begin{array}{l}\text { Relation to } \\
\text { Memory Transfer }\end{array}$ \\
\hline McConnell (1962) & $\begin{array}{l}\text { Report of successful transfer of learned memory in } \\
\text { planarian worms, using an associative learning } \\
\text { paradigm and cannibalism }\end{array}$ & Supports \\
\hline $\begin{array}{l}\text { Halas, James, \& } \\
\text { Stone (1961) }\end{array}$ & $\begin{array}{l}\text { Report of potential issues with memory association } \\
\text { paradigm from McConnell } 1962\end{array}$ & Ambiguous \\
\hline $\begin{array}{l}\text { Halas, James, \& } \\
\text { Knutson (1962) }\end{array}$ & $\begin{array}{l}\text { Report of failure to engage planarian worms in } \\
\text { associative learning paradigm from McConnell } 1962\end{array}$ & Challenges \\
\hline $\begin{array}{l}\text { Bennett \& Calvin } \\
\text { (1964) }\end{array}$ & $\begin{array}{l}\text { Report of failure to engage planarian worms in } \\
\text { associative learning paradigm from McConnell } 1962\end{array}$ & Challenges \\
\hline Babich et al. (1965) & $\begin{array}{l}\text { Report of memory transfer in rats, using a Skinnerian } \\
\text { learning paradigm and injection of neural tissue }\end{array}$ & Supports \\
\hline $\begin{array}{l}\text { Nissen, Røigaard- } \\
\text { Petersen, \& } \\
\text { Fjerdingstad (1965) }\end{array}$ & $\begin{array}{l}\text { Report of memory transfer in rats, using a } \\
\text { reinforcement learning paradigm and injection of } \\
\text { neural tissue; identification of reversal effect }\end{array}$ & Ambiguous \\
\hline Walker (1966) & $\begin{array}{l}\text { Report of confound of sensitization in protocol } \\
\text { presented in McConnell } 1962\end{array}$ & Challenges \\
\hline $\begin{array}{l}\text { Walker \& Milton } \\
\text { (1966) }\end{array}$ & $\begin{array}{l}\text { Report of confound of sensitization in protocol } \\
\text { presented in McConnell } 1962\end{array}$ & Challenges \\
\hline $\begin{array}{l}\text { Rosenblatt, Farrow, } \\
\text { \& Herblin (1966) }\end{array}$ & $\begin{array}{l}\text { Report of memory transfer in rats, using a Skinnerian } \\
\text { learning paradigm and injection of neural tissue }\end{array}$ & Supports \\
\hline $\begin{array}{l}\text { Rosenblatt, Farrow, } \\
\text { \& Rhine (1966a) }\end{array}$ & $\begin{array}{l}\text { Report of memory transfer in rats, using a Skinnerian } \\
\text { learning paradigm and injection of neural tissue; } \\
\text { replication of Babich et al. } 1965\end{array}$ & Supports \\
\hline $\begin{array}{l}\text { Rosenblatt, Farrow, } \\
\text { \& Rhine (1966b) }\end{array}$ & $\begin{array}{l}\text { Report of memory transfer in rats, using a Skinnerian } \\
\text { learning paradigm and injection of neural tissue }\end{array}$ & Supports \\
\hline Byrne et al. (1966) & $\begin{array}{l}\text { Report of failure to replicate memory transfer protocol } \\
\text { presented in Babich et al. } 1965\end{array}$ & Challenges \\
\hline $\begin{array}{l}\text { Røigaard-Petersen, } \\
\text { Nissen, \& } \\
\text { Fjerdingstad (1968) }\end{array}$ & $\begin{array}{l}\text { Report of memory transfer in rats, using a } \\
\text { reinforcement learning paradigm and injection of } \\
\text { neural tissue; modification to protocol in response to } \\
\text { Byrne et al. 1966; replication of reversal effect }\end{array}$ & Ambiguous \\
\hline Ungar (1970) & $\begin{array}{l}\text { Summary of reports of memory transfer, using a } \\
\text { reinforcement and Skinnerian learning paradigm and } \\
\text { injection of neural tissue }\end{array}$ & Supports \\
\hline $\begin{array}{l}\text { Goldstein, Sheehan, } \\
\text { \& Goldstein (1971) }\end{array}$ & $\begin{array}{l}\text { Report of failure to replicate memory transfer protocol } \\
\text { presented in Ungar } 1970\end{array}$ & Challenges \\
\hline $\begin{array}{l}\text { Krech \& Bennett } \\
\text { (1971) }\end{array}$ & $\begin{array}{l}\text { Report of failure to replicate memory transfer protocol } \\
\text { presented in Ungar } 1970\end{array}$ & Challenges \\
\hline $\begin{array}{l}\text { Ungar, Desiderio, \& } \\
\text { Parr (1972) }\end{array}$ & $\begin{array}{l}\text { Report of memory transfer in rats, using a } \\
\text { reinforcement learning paradigm and injection of } \\
\text { neural tissue }\end{array}$ & Supports \\
\hline
\end{tabular}

